\title{
Polaron bricklayers at work
}

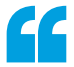

when we

realized that

the mechanism

behind this

reconstruction

was still

strongly

debated, we

conjectured

that it could

be related to

polarons
Surfaces can undergo reconstructions to minimize their free energy; the reconstruction is typically driven by the displacement of surface atoms or the transfer of electric charges between those atoms. However, polarons - excess charges trapped in the crystal lattice - can also be at the origin of surface reconstructions, as Cesare Franchini, Ulrike Diebold and colleagues report in Physical Review X.

Surface reconstructions modify the physical and chemical properties of surfaces; thus, they can provide a useful handle for the control of the surface properties. Polarons form more readily in the vicinity of surfaces, and they strongly influence the electronic and optical properties of materials, as well as their chemical reactivity. "Now, we have shown that they can also alter the atomic structure and guide a structural reconstruction of the surface," says Diebold.

In $\mathrm{TiO}_{2}$, a material of interest because of its many applications in catalysis, electronics and energyrelated devices, polarons can be introduced by

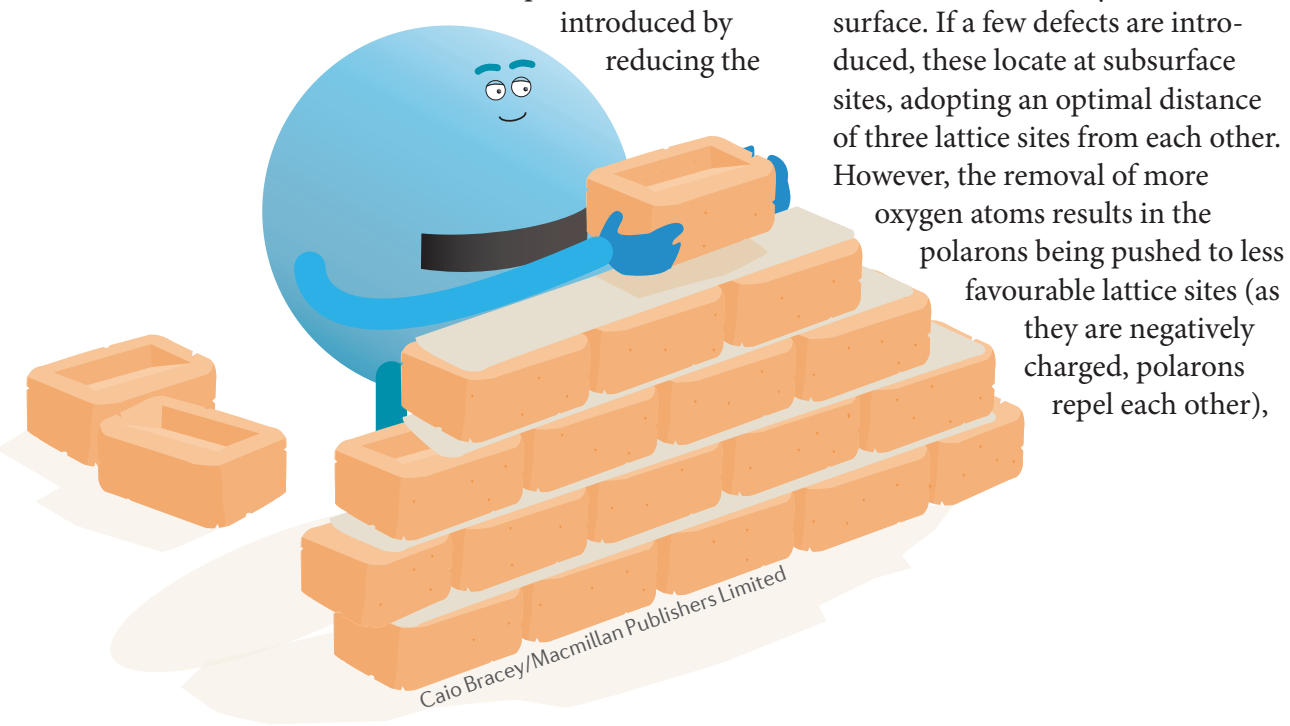

material through the addition of oxygen vacancies (in other words, by removing oxygen atoms). Upon reducing $\mathrm{TiO}_{2}(110)$ samples, a change in the surface reconstruction from $(1 \times 1)$ to $(1 \times 2)$ is observed - that is, the period of the surface structure is doubled in one direction.

"This work is the result of a theoretical and experimental collaboration that started about 5 years ago, during which we have characterized the basic physics of polarons in ionic materials such as $\mathrm{TiO}_{2}$," explains Franchini. "It has been known for a long time that the surface of $\mathrm{TiO}_{2}$ changes its structure when it is chemically reduced, and when we realized that the mechanism behind this reconstruction was still strongly debated, we conjectured that it could be related to polarons." Thus, the authors studied the changes in surface structure as a function of polaron concentration, using both experimental surface-science techniques and first-principles calculations.

When the sample is stoichiometric, it exhibits only the $(1 \times 1)$ surface. If a few defects are introduced, these locate at subsurface sites, adopting an optimal distance of three lattice sites from each other. However, the removal of more gen atoms results in the arons being pushed to less they are negatively harged, polarons repel each other), eventually leading to the appearance of $(1 \times 2)$ reconstructed stripes. The density of the stripes grows with further oxygen removal until they cover the entire surface. The structure of the $(1 \times 2)$ reconstruction has been a matter of debate until now, but, thanks to the precise determination of the position of the oxygen atoms afforded by atomic force microscopy, the researchers were able to confirm a previously proposed $\mathrm{Ti}_{2} \mathrm{O}_{3}$-based model.

Polaron formation is a ubiquitous phenomenon; thus, this mechanism is probably effective in many systems, and it will be possible to use it to tune materials properties in many oxide materials. "This will have an immediate impact on the catalytic properties of $\mathrm{TiO}_{2}$," comments Franchini. "Also, the ability of polarons to induce long-range structural modifications could be harnessed to optimize $\mathrm{TiO}_{2}$ based memristors, which are circuit elements with dynamic resistance influenced by structural changes in the device." Other objectives for future research include the study of the relationship between the presence of polarons and the catalytic properties of the surface, the control of polarons by external electric fields and the study of polaron-mediated reconstructions at the interface between different materials.

Giulia Pacchioni ORIGINAL ARTICLE Reticcioli, M. et al. Polarondriven surface reconstructions. Phys. Rev. X 7 , 031053 (2017) 